\title{
Numerical modeling of particle migration in granular soils
}

\author{
I. G. Tejada \& L. Sibille \& B. Chareyre \\ Université Grenoble Alpes, Grenoble-INP, CNRS, 3SR Laboratory, Grenoble, France \\ E. Vincens \\ University of Lyon- Ecole Centrale de Lyon, CNRS, LTDS, Lyon, France
}

\begin{abstract}
Suffusion is the process of internal erosion where fine particles migrate under water seepage through a coarser soil matrix. Relevant models of suffusive phenomena must reproduce the poromechanical effects that result from the two-way coupling between the deformation of the solid matrix, the fluid pressure and the flow. In this work, an advanced computational method is used to study the particle migration in granular soils. The so called coupled Discrete Element Method - Pore scale Finite Volume (DEM-PFV) is based on a microscopic hydromechanical approach. It couples the discrete element method that solves the equations of motion for the solid fraction, with a PFV method that solves the fluid flow equations. We use this method to study particle transport through coarser granular assemblies that do not evolve with time. These simulations allow us to obtain, for different cases, the parameters to include in a general advection-dispersion equation (ADE). We paid particular attention to the role played by the intermittent formation of blockages of transported particles in the constrictions of the granular assembly. These temporary and collective trapping events change local fluid flows and affect the particle transport on short time or length scales. As the transport time between consecutive blockages and the duration of blockages have exponential decays, sink and source terms can be added to the ADE.
\end{abstract}

\section{INTRODUCTION}

The description of the process of internal erosion in soils has attracted a lot of attention in geotechnical engineering since it is one of the most common cause of failure of earth dams and dikes. Moreover, its consequences may be especially dangerous since internal erosion is hard to detect in advance.

Internal erosion is referred to as the migration of soil particles caused by water seepage. It includes concentrated leak erosion (when, for instance, a continuous pipe develops in the soil), contact erosion (produced at the interface between fine and coarse soil layers) and suffusion (the migration of the fine fraction that happens in the bulk of the soil). In all these cases, particle transport takes place and particularly in suffusion leading to a decrease of the mechanical properties of the soil.

Suffusion is a complex phenomenon owing to the diversity of the involved mechanisms (?). The potential for internal stability is determined by the particle size distribution of the material, while the onset and the development of suffusion are determined by the hydromechanical behavior of the system.

In order to understand the highly non-linear behav- ior of the system when suffusion takes place, the complex interplay between eroded particles, coarse skeleton and carrier fluid must be well reproduced. How the features of each phase determine the interactions between them is something that must be investigated.

Suffusion comprises at least three microscopic phenomena: the detachment of fine solid particles from the solid matrix, the transport of these particles as a consequence of the fluid flow and, eventually, the filtration downstream of these particles at different points within the soil.

The detachment of fine particles can be due to local changes in the fluid flow that introduce perturbations in the local structures, but also by degradation or ageing processes. The filtration is something that may happen at different sites within the soil (throats, surfaces, cracks) when fluid pressure and friction, or other forces (van der Waals, electrical, chemical) (?), hold particles which were previously eroded and conveyed. The transport is produced by viscous stresses and local hydraulic pressure gradients that make particles move through a complex and dynamic geometry. Even when from a macroscopic point of view, the fluid flow is supposed to be homogeneous, the reality is that the geometrical randomness of the soil at 
the grain level induces randomness in the flow field and stochastic trajectories in the transported particles. Indeed the reality is that even in quite homogeneous systems, there are preferential flow paths $(?, ?)$ and the transport of particles is not well described with simple transport models.

For the sake of simplicity, we studied the case in which there is a coarse granular assembly that is completely separated from a set of erodable particles (which are finer but still comparable to the typical size of pores of the assembly). The assembly of coarse particles is fixed while the set of fine particles moves through it. The fluid flows through long and thin tortuous passages of varying cross sections created by the fixed coarse skeleton and the moving fluidized particles. A low value of the Reynolds number is expected, so the flow is truly laminar and the mean flux (at given hydraulic pressure drop and fluid viscosity) depends on the permeability, which is in turn a consequence of the porosity and the complexity of the structure. When the concentration of transported particles increases, the porosity is reduced and the pore throat openings are dramatically reduced. The consequence is that the permeability, and hence the fluid flux, decreases. With regard to the transported particles, as the concentration increases, collisions are more frequent and the mean free path length (the displacement between two consecutive collisions) is reduced. These interactions affect the spread of the particles and the average displacement after a given time. However at some points, other interesting events may take place. For example, some particles may clog a constriction and thus avoid others to pass through it, altering not only the particle transport but also the fluid flow (Fig. 1 a). Moreover, further moving particles may collide and break down the structures supporting these blockages.

Therefore, particle transport is a complex process fully determined by events that occur at the pore level. In this work, an advanced micro-hydrodynamical computational method (DEM-PFV) is used to study the particle migration in granular soils. First, we explain how particle transport can be modeled from a continuous approach based on advection and dispersion mechanisms. In particular we point out the issues related to such a description and due to the intermittent formation and destruction of blockages that can affect the macroscopic features of the particle transport. In a second part, the micro-hydromechanical numerical model of particle transport is briefly described. Its capability to reproduce the formation of collective blockages of transported particles in the interstitial space of the porous medium is discussed. Then data resulting from direct numerical simulations of particle transport are extracted and used to calibrate the continuous model. Finally continuous and microhydromechanical models are compared.

\section{MODELING PARTICLE TRANSPORT}

The saturated soil is viewed as a three-phase medium consisting of (1) a solid coarse skeleton that does not evolve in time, (2) some fluidized grains that are within the pores of the coarse skeleton and (3) a fluid that fills the pores and flows through the porous structure according to hydraulic gradients. The fluid flow may convey the fluidized grains through the coarse skeleton. Then, the total volume $V$ of a Representative Elementary Volume (REV) of soil is equal to the sum of the volumes of the coarse skeleton $V_{\mathrm{c}}$, the fluidized particles $V_{\mathrm{p}}$ and the fluid $V_{\mathrm{w}}$. As neither $V$ nor $V_{\mathrm{c}}$ change in time, the void space $V_{\mathrm{v}}=V_{\mathrm{p}}+V_{\mathrm{w}}$ and the porosity $\Phi=V_{\mathrm{v}} / V$, remain invariable. The concentration of transported particles is defined as $c=V_{\mathrm{p}} / V_{\mathrm{v}}$ or as $C=V_{\mathrm{p}} / V$, with $C=c \Phi$.

The velocity field of the water flow is $\boldsymbol{v}_{\mathrm{w}}$ and the associated volume discharge $\boldsymbol{q}_{\mathrm{w}}=(1-c) \Phi \boldsymbol{v}_{\mathrm{w}}$. The velocity field is obtained after applying the mass balance for the fluid phase:

$\nabla \cdot q_{\mathrm{w}}=\mathbf{0}$.

According to Darcy's law, $\boldsymbol{q}_{\mathrm{w}}=-\frac{1}{\mu} \boldsymbol{\kappa} \boldsymbol{\nabla} p$, being $\boldsymbol{\kappa}$ the permeability tensor, $\mu$ the dynamic viscosity of the fluid and $p$ the fluid pressure. Therefore, the flow equation reads: $\boldsymbol{\nabla} \cdot(\boldsymbol{\kappa} \nabla p)=\mathbf{0}$

Similarly, the mass balance for the fluidized solid phase is expressed as

$\frac{\partial C}{\partial t}+\boldsymbol{\nabla} \cdot \boldsymbol{j}=\mathbf{0}$

where $j$ is the total particles flux. The usual paradigm of particle transport states that $j$ is produced after the combination of advection and dispersion mechanisms, so that $\boldsymbol{j}=\boldsymbol{j}_{\text {advection }}+\boldsymbol{j}_{\text {dispersion }}$.

Advective particle transport models the fact that the fluid flow establishes some prevailing directions. In other words the center of mass of the particles cloud moves parallel to the macroscopic fluid flow and $j_{\text {advection }}=\boldsymbol{v}_{\mathrm{p}} C$. In general, the higher the fluid flux, the longer the distance the center of mass will move, since drag, viscous and pressure forces will be stronger. Then, the average velocity of the traveling particles is a fraction of the fluid velocity $\boldsymbol{v}_{\mathrm{p}}=\chi \mathbf{v}_{\mathrm{w}}$, with $\chi \in[0.0,1.0]$. For a given porous medium, the actual value of $\chi$ depends on the size of the particles with respect to that of pores, on their shape, on the interparticle friction and on the value of $c(?, ?, ?, ?)$. Indeed, if the transported particles are much smaller than the characteristic sizes of pores and throats, particles will closely follow the streamlines of the flow and $v_{\mathrm{p}}$ will approximate $v_{\mathrm{w}}$. Conversely, when the size of transported particles is comparable to the size of pores and throats, the relative flow between transported particles and the carrier fluid is very significant since the interaction of the fluidized particles with the coarse 


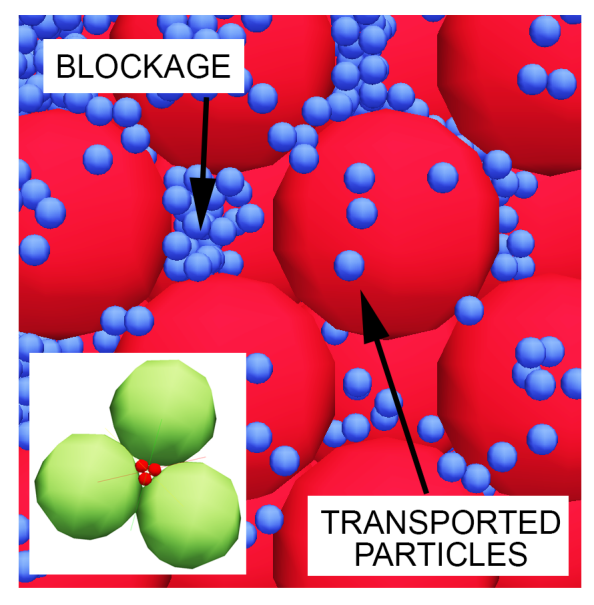

(a)

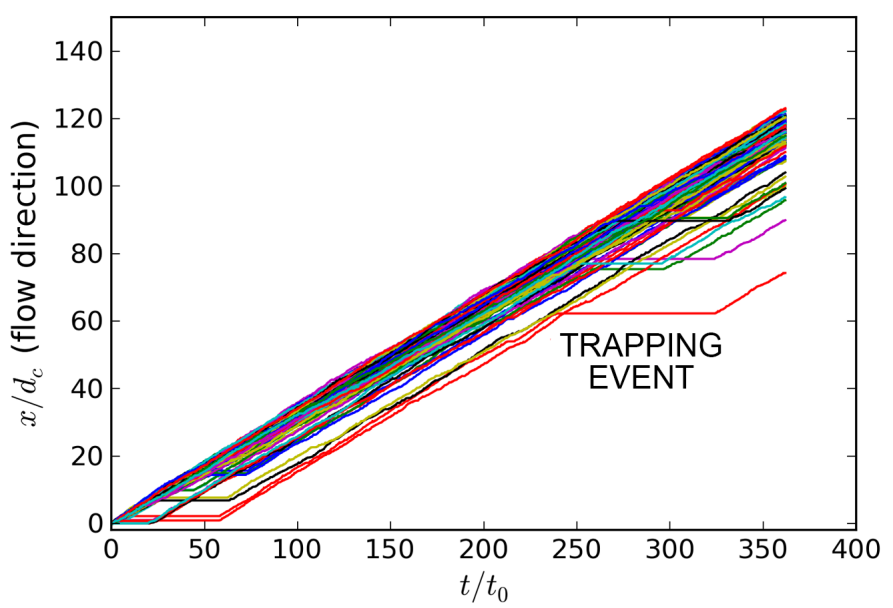

(b)

Figure 1: Numerical experiments performed with a hydro-micromechanical model (?). (a) Intermittent formation of blockages in the constrictions (the inset represents the arch formed by three fine frictionless particles that originates a blockage). (b) Statistical sample of particle displacements (in the direction of the water flow) versus time. Horizontal intervals represent periods in which the corresponding particle was trapped (i.e. it did not change its position with time).

skeleton or between themselves is different from of the fluid flow.

Dispersive particle transport accounts for the fact that in a frame of reference that moves with the convective velocity, the cloud of transported particles does not remain invariable with the transport because each particle undergoes its own history of collisions. In contrast to advection, dispersion is random in nature (it does not necessarily follow the direction of the fluid flow) and depends on the concentration (the transport evolves from regions of high concentration to low concentration, with an equilibrium state of uniform concentration). Under the standard dispersion paradigm, the dispersive transport of solid particles is characterized by the Fourier-Fick's law

$\boldsymbol{j}_{\text {dispersion }}=-\boldsymbol{D} \nabla C$

where $D$ is the hydrodynamic dispersion tensor. As the dispersion is a consequence of the fluid-particle interactions, $\boldsymbol{D}$ is related to $\boldsymbol{v}_{\mathrm{w}}$ via the dispersivity tensor. For example, an expression for isotropic media has been proposed (?):

$\boldsymbol{D}=d_{\mathrm{t}} v_{\mathrm{w}} \boldsymbol{I}+\left(d_{1}-d_{\mathrm{t}}\right) \frac{\boldsymbol{v}_{\mathrm{w}} \otimes \boldsymbol{v}_{\mathrm{w}}}{v_{\mathrm{w}}}$,

where $\boldsymbol{I}$ is the identity tensor and $d_{1}$ and $d_{\mathrm{t}}$ are the two parameters of the model, denoting, respectively, the longitudinal and the transversal dispersivity.

After including advection and dispersion terms, the transport equation reads

$\frac{\partial C}{\partial t}+\nabla \cdot\left(\boldsymbol{v}_{\mathrm{p}} C\right)-\boldsymbol{\nabla} \cdot(\boldsymbol{D} \nabla C)=\mathbf{0}$,

The advection - (Fickian) dispersion equation ADE comes from a phenomenological approach to particle transport but it can be understood by focusing on the paths of the fluidized particles. Indeed, particle tracking allows statistical samples of particle trajectories over different length and time scales to be generated. If one of these samples $\left\{\boldsymbol{x}_{1(t)} \cdots \boldsymbol{x}_{1(t)}\right\}$ meets the requirements for normal transport, after a time $t$ the sample mean will be located at $\overline{\boldsymbol{x}}_{(t)}=\left\langle\boldsymbol{x}_{(t)}\right\rangle=\boldsymbol{v}_{\mathrm{p}} t$, while the centered mean-squared displacement, defined as $M S D=\sigma^{2}=\left\langle\left(\boldsymbol{x}-\boldsymbol{v}_{\mathrm{p}} t\right)^{2}\right\rangle$ will grow linearly in time, i.e. $\sigma^{2}=2\left(D_{I}+D_{I I}+D_{I I I}\right) t\left(D_{I}\right.$, $D_{I I}$ and $D_{I I I}$ being the three principal components of the hydrodynamic dispersion tensor). Therefore, finding linear relationships between the sample mean and the sample variance with time is a guarantee for normal transport and allows for the identification of the values of the advective velocity and the dispersion coefficients. However this is not always the case. From the point of view of statistical mechanics, Fickian or normal dispersive transport is conceived as the macroscopic manifestation of an underlying "microscopic" uncorrelated, Gaussian stochastic process, i.e., a Brownian random walk. The normal dispersion mechanism is then an outcome of the central limit theorem. In some circumstances or for some length or time scales, these requirements are not met and the ADE equation fails. For example, on very short time scales (typically when length scales are comparable to the mean free path or to the transit between pores) the motion of the particles is strongly autocorrelated and the ADE fails. But there may be other reasons to find anomalous transport on longer length / time scales. Among these reasons, multiscale heterogeneities of porous media are found to be one of the reasons for anomalous transport $(?, ?, ?, ?$, ?). For example, in the case of porous media which have high flux pipes (i.e. pipes that are wider or less tortuous than the average) and tend to be spatially continuous at all scales, particles traveling faster or slower than the mean are much more likely to keep the same property over a large distance. But even in quite 
homogeneous systems, the existence of preferential flow paths may lead to anomalous transport $(?, ?)$. Indeed in random fractured networks subtle features of the steady flow-field distribution through the network are behind time-dependent anomalies (?). Yet another reason for anomalous transport is given by traps: whatever mechanism that retains the fluidized particles for a while. The frequency and duration of trapping events establish the lengths and time scales in which transport is normal. These scales can be much longer than those corresponding to the typical free path or the pore size since they depend on the duration of blockages and on the time or length that particles travel without stopping. Therefore, trapping events may have significant implications for particle transport since on short time or length scales (shorter than the lifetime of trapping events and transport periods) the ADE fails to explain the evolution of the concentration of fluidized particles.

In this work, we have focused on one of these trapping events: the intermittent formation of blockages in the constrictions of the porous skeleton. Particles may be directly intercepted when their sizes are smaller than the constrictions but they can also participate in collective clogging events, when a temporary arch is formed. While the arch exists, other particles can be stopped upstream and form a blockage. Obviously the constrictions size distribution $\operatorname{CSD}(?, ?)$ of the coarse skeleton plays a crucial role, since it fosters or hampers the formation of blockages. But also the concentration of transported particles is decisive since the clogging of a given constriction is basically a matter of the probability of the presence of particles passing through it $(?, ?)$. Indeed the formation of arches is hardly affected by the driving force (here the fluid flow), as it has been proved in the case of silo clogging (?). We have checked how, under conditions of constant hydraulic gradient, blockages appear and disappear in the constriction of a macroscopically homogeneous coarse skeleton. Then, the trajectories of transported particles are well described as successions of waiting (trapping events) and walking periods. During the walking periods the motion of particles is well reproduced by Eq. 5 but the possibility of waiting times adds complexity to the evolution of $C$. This kind of motion is known as a Levywalk (?) in the context of continuous time random walks CTRW $(?, ?)$. In CTRWs the probability distribution functions (PDFs) of the duration of blockages and displacements state whether or not the ADE paradigm is valid and, if yes, when it is. If these PDFs have finite second moment, then the central limit theorem applies, and on a time scale longer than the typical mean times, the normal transport is recovered. In other words, conditions for normal transport are met when the memory effect of blockages has been completely erased.

The numerical experiments we performed show that these PDFs fit exponential laws ${ }^{1}$ (Fig. 2), reinforcing the idea that homogeneous Poisson processes are behind the formation and destruction of blockages (?). The exponential time constants that describe these laws are $\tau_{\mathrm{f}}$ for particle filtration (the lifetime of particle displacements between consecutive blockages) and $\tau_{\mathrm{r}}$ for particle release (the lifetime of blockages). Accordingly, filtration and release terms (sink and sources) can be added to Eq. 5. Then it seems necessary to distinguish between retained particles $S$ and particles in motion $T$, in such a way that $C=S+T$. Thus Eq. 5 evolves towards a system of EDPs:

$$
\begin{aligned}
\frac{\partial T}{\partial t}+\nabla \cdot\left(\boldsymbol{v}_{\mathrm{p}}^{*} T\right)-\nabla \cdot\left(\boldsymbol{D}^{*} \nabla T\right) & =-\frac{T}{\tau_{\mathrm{f}}}+\frac{S}{\tau_{\mathrm{r}}} \\
\frac{\partial S}{\partial t} & =+\frac{T}{\tau_{\mathrm{f}}}-\frac{S}{\tau_{\mathrm{r}}},
\end{aligned}
$$

where $\boldsymbol{v}_{\mathrm{p}}^{*}$ and $\boldsymbol{D}^{*}$ are the actual values of the advective velocity and the hydrodynamic dispersion tensor that exclusively apply when the particles are being conveyed (between two consecutive blockages). When $t \gg \tau_{\mathrm{f}}, \tau_{\mathrm{r}}$, the features modeled by Eq. 5 are recovered (with $\left.\boldsymbol{v}_{\mathrm{p}}=\left(\tau_{\mathrm{f}} /\left(\tau_{\mathrm{r}}+\tau_{\mathrm{f}}\right)\right) \boldsymbol{v}_{\mathrm{p}}^{*}\right)$, and there is no need to use Eq. 6.

\section{NUMERICAL MICRO-HYDROMECHANICAL MODELING OF INTERNAL EROSION}

A key feature in particle transport through porous materials is the two-way coupling between the motion of the particles and the fluid flow. In order to understand this two-way coupling, a detailed description of the stress exchange between solid grains and fluid is required. On the one hand, the fluid flow exerts forces on the coarse skeleton and on fluidized particles. These forces, caused by viscosity and pressure gradients, may convey the particles and eventually form arches or destroy those that were previously formed. On the other hand, the motion of the particles creates a dynamically changing domain for the fluid flow. These two effects are combined when hydraulic pressure gradients generate fluid and particles flows through a coarse skeleton.

As all the macroscopic features of the transport arise from complex events that occur at the pore level, it is interesting to use models capable of reproducing the time evolution of fluidized grains and the fluid flow at that level. While the movement of solid grains can be efficiently modeled with the Discrete Element Method (?), viscous fluid computations in dynamically changing domains present formidable computational challenges.

\footnotetext{
${ }^{1}$ The bad fitting of the trapped time for the lowest values of $C$ is probably due to an insufficient size of the statistical sample (a few trapping events were observed during the simulation time).
} 


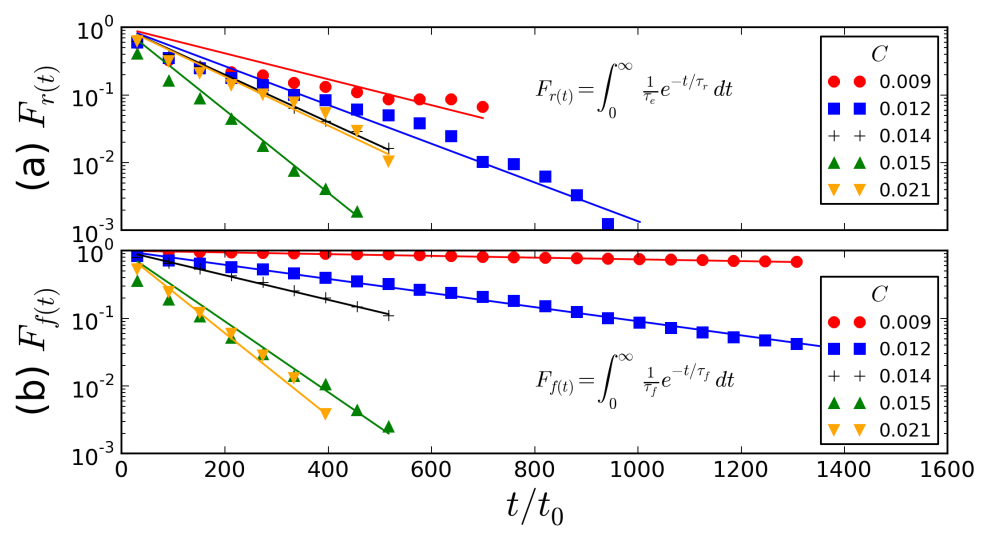

Figure 2: Cumulative distribution functions of trapping (a) and traveling times (b). The markers show the results of numerical experiments and the dotted lines are the least squares approximations to an exponential decay (?).

\subsection{The DEM-PFV method}

Different strategies have been adopted to combine the DEM with a modeling techniques for the fluid part of the problem. Among them, we find Microscale Stokes Flow Models in which the Stokes equations are numerically solved at the subpore scale with Finite Element or Lattice-Boltzmann methods (e.g. (?)). We can also find Continuum-Discrete Darcy's Flow Models, in which coarse grid computational fluid dynamics methods are used for the fluid and simplified semiempirical models (based on Darcy's law) for the interaction between solid particles and fluid. The PoreNetwork Flow Modeling approaches belong to a third class. They are based on a simplified representation of porous media (mostly developed to predict the permeability of materials from microstructural geometry or to study multiphase flow effects).

This work is based on this latter technique. However, the proposed method has been conceived to handle dynamically changing domains for the fluid flow. The so-called DEM-PFV (?, ?) is a microhydromechanical approach to the problem that couples the DEM with a pore-scale finite volume method. This method applies for Stokes flows (i.e. at the subpore scale, the fluid flow is governed by Stokes equations, which express fluid mass and momentum conservation at small Reynolds number) of incompressible and Newtonian fluids. At a given time, the actual geometry of the packing defines an open network of connected voids (Fig. 3). The method uses a regular triangulation (?) and its dual Voronoi graph to discretize the void space. The flux between two adjacent tetrahedra is computed according to the local geometry of the common facet and the difference of water pressures in the pores (in analogy to the Kozeny-Carman derivation). This is a generalization of the Hagen-Poiseulle equation that is consistent with Stokes equations. On the other hand, the total force exerted by the fluid on a fine flowing particle results from the pressure and viscous stress acting at the surface of the particle. The method is fully coupled in such a way that the change of volume of a tetrahe- dron takes into account the particle movements and is equal to the net fluid flow, and the particles move according to the forces (including viscous and hydraulic pressure forces exerted by the fluid flow).

\subsection{Numerical experiments of particle transport in crystalline granular assemblies}

We have carried out numerical experiments of particle transport through crystalline granular assemblies. The purpose of these experiments was to generate statistical samples of particle trajectories and trapping events that allow us to set up a continuous model for transport and measure its parameters.

We have used the DEM-PFV, implemented in YADE (?), to model particle transport in fixed crystalline granular assemblies. On the length and time scales in which the transport is normal (when $v_{\mathrm{p}} \propto t$ and $\sigma^{2} \propto t$ ), after the analysis of particle paths we can get the parameters to include in a general ADE. On shorter scales we can pay attention to the PDFs of trapping and walking times.

Regarding the DEM, Hookean and frictionless contact are used to model interactions between the particles. For the coarse skeletons, we used crystalline and quasi-crystalline structures made of equal sized spherical particles of diameter $d_{\mathrm{c}}=5 \cdot 10^{-3} \mathrm{~m}$. Quasicrystalline structures were generated by slightly enlarging the unitary cells of crystalline structures and randomly moving the particles. In particular we report data of particle transport through quasi Simple Cubic ( $\mathrm{SC}^{*}$ ) and quasi and perfect face-centered cubic (FCC* and FCC, respectively). $\mathrm{SC}^{*}$ structure is characterized for producing continuous and straight pipes. Its porosity was $\phi_{\mathrm{SC}^{*}} \simeq 0.53$ (instead of that of a perfect SC, i.e. $\phi_{\mathrm{SC}} \simeq 0.48$ ). The porosities of $\mathrm{FCC}^{*}$ and $\mathrm{FCC}$ were, respectively, $\phi_{\mathrm{FCC}} \simeq 0.30$ and $\phi_{\mathrm{FCC}} \simeq 0.26$. FCC is the densest possible packing obtainable with equal-sized spheres. In such a case, the geometrical configuration of particles creates the smallest possible constriction size. A random close packing of equal size spheres (RDN) was also used but just in order to measure the advective and fluid velocities, but not other transport parameters. The in- 


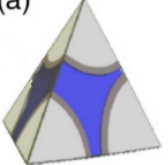

(b)
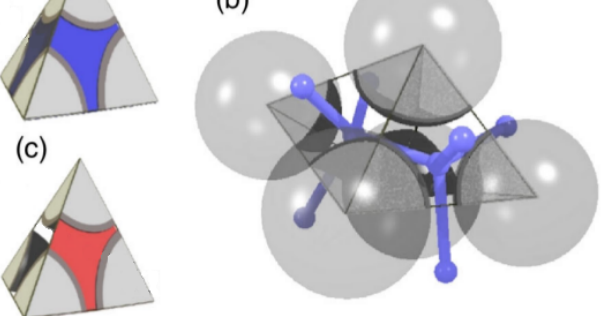

Figure 3: (a) Volume of fluid in a pore, (b) adjacent pores and local connections, (c) fluid domain of pore contour (?).

terest is that in this case the structure is random and its porosity lies between those of $\mathrm{SC}^{*}$ and $\mathrm{FCC}^{*}$ structures. In all the cases the coarse skeleton is fixed so the domain for the fluid flow exclusively changes as a consequence of the relocation of the fluidized particles.

In the structures that we used the CSD is controlled and, as they are macroscopically homogeneous, these structures do not introduce any multiscale heterogeneity that can affect the particle transport. In other words, all the possible reasons for anomalous transport will be exclusively due to the role played by the fluidized particles, and especially, to the blockage events. Furthermore, the use of crystalline assemblies in association with periodic boundary conditions allows to use fewer particles in the system. Thus, we can model situations with a considerably high concentration of fluidized particles. Typically we used $2 \times 2 \times 2$ unit cells (i.e. 8 coarse particles for the SC and 32 for the FCC).

Concerning the fluidized grains, we used equalsized spherical particles of diameter $d_{\mathrm{f}}=d_{\mathrm{c}} / 8$ that were randomly introduced in the voids of the coarse skeleton. $d_{\mathrm{f}}$ is chosen below the maximum value that a frictionless particle can have in order to pass through the smallest possible constriction found in FCC media $\left(d_{\mathrm{c}}(2 \sqrt{3}-1)\right)$, so that the fluidized particles cannot been trapped alone anywhere but three of them or more can form an arch in the smallest possible constrictions. Once an arch appears, other particles can be trapped upstream, forming a blockage.

For the boundary conditions in terms of solid and fluid, a constant hydraulic pressure gradient and periodic boundary conditions for fluidized particles and fluid flows have been imposed. By changing the total amount of fluidized particles, we observe particle transport for different values of $C$.

Gravity is not considered in the model to avoid the effects of particle sedimentation.

\section{RESULTS AND DISCUSSION}

Hereafter, we report the values of the velocity and dispersion in the direction of the fluid flow $(-\nabla \boldsymbol{p})$, in Tab. 1. The cases examinated differ in the kind of crystalline structure and the concentration. The data are normalized as follows. We use $t_{0}=d_{\mathrm{c}} / v_{0 \mathrm{w}}$ as a
Table 1: Values of transport parameters to use in Eq. 6 obtained from DEM-PFV simulation.

\begin{tabular}{lr|rrrrr}
\multicolumn{2}{c}{ Case } & & \multicolumn{5}{c}{ Transport parameters } \\
\hline Structure $(\phi)$ & $C$ & $v_{\mathrm{p}} / v_{\mathrm{w}}$ & $v_{\mathrm{p}}^{*} / v_{\mathrm{w}}$ & $P e^{*}$ & $\tau_{\mathrm{f}} / t_{0}$ & $\tau_{\mathrm{r}} / t_{0}$ \\
\hline $\mathrm{SC}^{*}(0.53)$ & 0.005 & 0.018 & 0.018 & 1.8 & $\infty$ & - \\
$\mathrm{SC}^{*}(0.53)$ & 0.045 & 0.042 & 0.042 & 2.5 & $\infty$ & - \\
$\mathrm{SC}^{*}(0.53)$ & 0.091 & 0.111 & 0.111 & 2.3 & $\infty$ & - \\
\hline FCC $^{*}(0.30)$ & 0.000 & 0.047 & 0.047 & 24.1 & $\infty$ & - \\
FCC $^{*}(0.30)$ & 0.005 & 0.055 & 0.055 & 25.2 & $\infty$ & - \\
FCC $^{*}(0.30)$ & 0.009 & 0.057 & 0.057 & 29.7 & $\infty$ & - \\
FCC $^{*}(0.30)$ & 0.012 & 0.061 & 0.061 & 26.7 & $\infty$ & - \\
\hline FCC $(0.26)$ & 0.009 & 0.098 & 0.099 & 14.7 & 3380 & 227 \\
FCC $(0.26)$ & 0.012 & 0.076 & 0.104 & 13.8 & 416 & 152 \\
FCC $(0.26)$ & 0.014 & 0.069 & 0.092 & 22.0 & 239 & 124 \\
FCC $(0.26)$ & 0.015 & 0.059 & 0.100 & 15.5 & 205 & 180 \\
FCC $(0.26)$ & 0.021 & 0.043 & 0.100 & 11.0 & 71 & 120 \\
\hline
\end{tabular}

reference time, being $v_{0 \mathrm{w}}$ the velocity of the fluid in a clean structure (i.e. when $C=0$ ). The Péclet number $P e^{*}$ is the ratio of the rate of advection of particles to the rate of dispersion of particles, so that $P e^{*}=v_{\mathrm{p}}^{*} d_{\mathrm{c}} / D$. In other words, the value of the $D$ to include in Eq. 6 is $D=v_{\mathrm{p}}^{*} d_{\mathrm{c}} / P e^{*}$.

The numerical experiments show (Fig. 4) how as the porosity is reduced or the concentration is increased, both the fluid flux and the advective velocity are reduced. However the exact reverse is true for the ratio of the actual advective velocity to the velocity of the fluid: it increases when $C$ does, but decreases when $\phi$ increases. It is due to the fact that when there are more fluidized particles in the voids, the tortuosity of the fluid increases (there are more and narrower passages for the fluid) while that of the particle paths is reduced (the free path is shorter). In other words, the viscosity of the complex fluid (fluid plus transported particles) is increased so that the flux is reduced and the flow becomes more laminar.

With the chosen fluidized particles, $\mathrm{SC}^{*}$ and $\mathrm{FCC}^{*}$ structures do not allow arch formation in the constrictions. As any other trapping event is possible, on a length scale longer than the pore size the motion of the particles is not autocorrelated. Therefore the normal advection - dispersion paradigm is recovered soon and the dispersion coefficient can be measured.

In contrast, in FCC structures, an intermittent formation of blockages in the constrictions has been observed. The duration of the blockages and especially the time that particles move between consecutive blockages is determined by the concentration. These times can be longer or much longer than the to- 


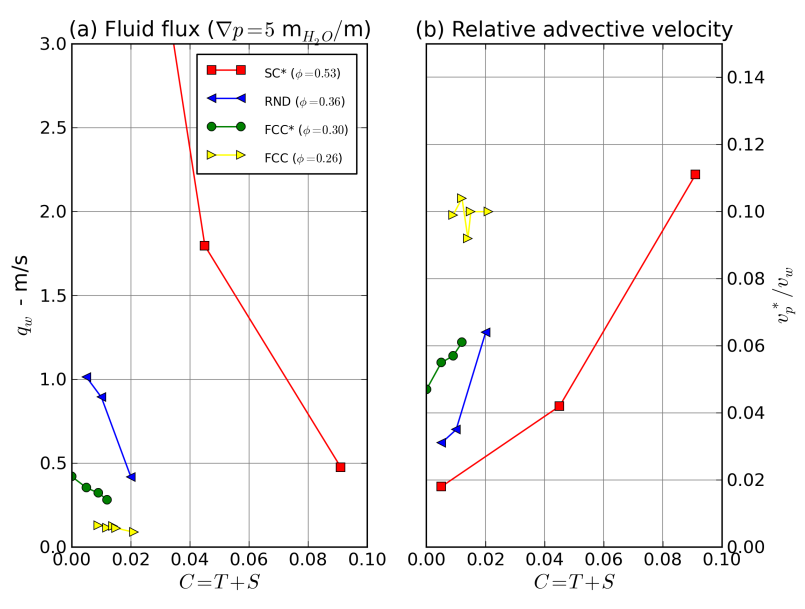

Figure 4: Particle and fluid velocities obtained from DEMPFV simulations of particle transport for different structures and concentrations of transported particles subjected to the same hydraulic pressure gradient. (a) Fluid flux (i.e. the permeability). (b) Ratio between the actual advective velocity of the transported particles and the velocity of the fluid.

tal observation time, which gives an idea on the time scales their effects on the transport are erased. As they seem to follow exponential decays, the conditions for normal dispersion on a longer time scale are ensured. On the time scale of our experiments, we can use Eq. 6 with the values included in Tab. 1. For example, the evolution of $C$ obtained with the DEM-PFV and compared to Eq. 6 for the case of an FCC structure is shown in Fig. 6. More precisely, the statistical distribution of the displacements of particles with respect to their initial positions after a time of $1150 t_{0}$ in the case of a FCC structure with a uniform value of $C=0.012$ are given.

However the exponential time constants for particle filtration $\tau_{\mathrm{f}}$ and release $\tau_{\mathrm{r}}$ strongly depend on the concentration of transported particles (Fig. 5). Both of them become similar for the higher values of $C$ and $\tau_{\mathrm{f}}$ tends to $\infty$ when $C \rightarrow 0$. The pronounced dependency of filtration and erosion with concentration (as well as the permeability) have important implications in the evolution of $C$ in transient regimes since it makes Eqs. 1 and 6 to be highly non-linear. For example a rough fitting of the filtration data to a power-laws gives $\tau_{\mathrm{f}}=\tau_{(0) \mathrm{f}} C^{-\alpha}=\tau_{(0) \mathrm{f}}(T+S)^{-\alpha}$. With such a law, the sink term in Eq. $6,-T / \tau_{\mathrm{f}}$, would be proportional to $-(T+S)^{\alpha} T$. This term, together with another expression $\tau_{\mathrm{r}}=f_{(T+S)}$, leads to complex transient solutions on the short term regime. On the other hand, the corresponding ratio of the exponential times for erosion and filtration for each $C$ also gives the equilibrium ratio of transported to filtered particles expected in an equilibrium state. If the system were out of the equilibrium, it will tend to it.

\section{CONCLUSIONS}

We have used a micro-hydromechanical model that is able to reproduce micromechanical events that oc-

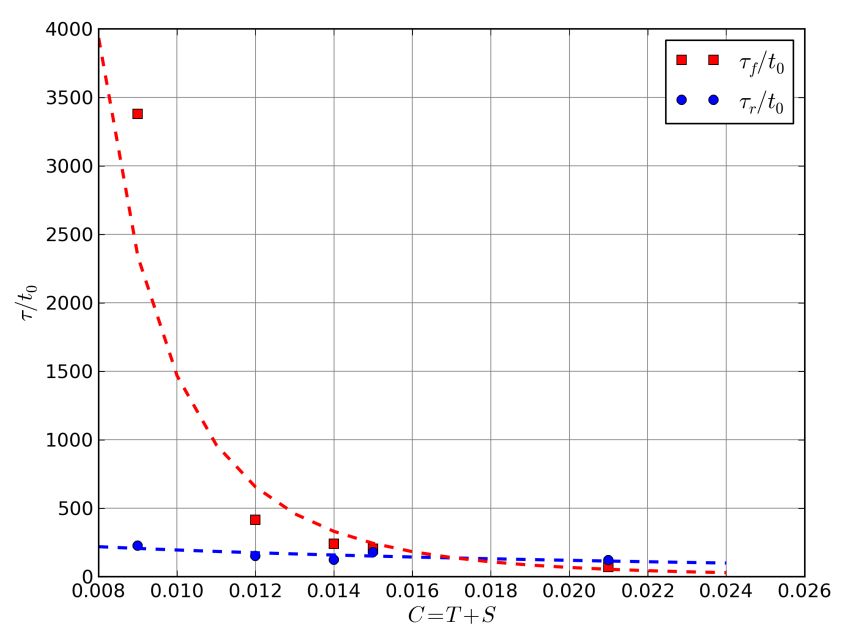

Figure 5: Exponential times of erosion and filtration in a FCC structure for different values of $C$ obtained from DEM-PFV simulations.

cur when solid particles are carried by a Stokes flow through a porous structure. We focus on the role played by the temporary blockages that are formed in the constrictions of the assembly in some circumstances. Both the duration of particle displacements and of blockages follow exponential decays, with exponential factors that strongly depend on the concentration of particles. Similarly, the permeability, the advective velocity and the dispersion of particles depend on the concentration of transported particles. Consequently, the equations for particle transport are highly non-linear. All this leads to a complex short term for particle transport much larger than the time characterizing free paths or transits between pores. On this time scale a system of equations that considers traveling and stopping fractions can be used. Once the effect of blockages is erased (on longer time scales), the classical advection - dispersion equation can be used.

\section{ACKNOWLEDGEMENT}

This work has been supported by the LabEx Tec21 (Investissements d'Avenir - grant agreement nANR11-LABX-0030). Acknowledgement is also given to the regional federative structure VOR (Vulnérabilité des Ouvrages aus Risques). 


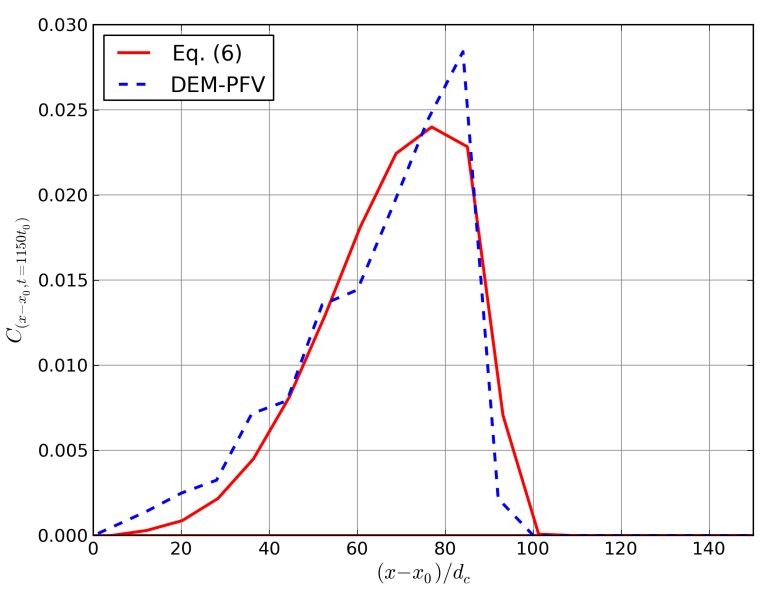

Figure 6: Comparison of the evolution of the concentration of fluidized particles in a FCC structures when $C=0.012 . x-x_{0}$ denotes the displacement of particles with regard to their positions at $t=0$. 